\title{
EL MATERIAL DIDÁCTICO QUE APORTA EL ALUMNO. UNA EXPERIENCIA DE CONSTRUCCIÓN SIGNIFICATIVA DEL APRENDIZAJE A TRAVÉS DE PROYECTOS
}

\author{
Teresita E. Terán, Augusto Nascimbene \\ Universidad Nacional de Rosario, Argentina \\ teresitateran@hotmail.com
}

En este trabajo se destaca el valor del Proyecto como eje del aprendizaje. Esta posición se basa en la existencia de dificultades en la comprensión de los conceptos matemáticos, desajustes entre los Significados Institucionales y el Significado Personal construido por el alumno y la posibilidad de diseñar algunas metodologías alternativas a la enseñanza tradicional en la Universidad que faciliten el aprendizaje. La experiencia se desarrolló durante el año 2014 en las comisiones de Bioestadística, en la Facultad de Ciencias Veterinarias de la U.N.R. Se describe la trayectoria didáctica en temas de Estadística Descriptiva, Probabilidad y breves nociones de Inferencia. Entre los resultados se pudo observar que el método de proyectos mejora sustancialmente la asimilación de diversos contenidos estadísticos, siendo la "formulación y comunicación en terminología estadística" y "la validación y la expresión de conclusiones en términos del problema”, siendo los más ausentes en la construcción de los conceptos.

\section{PLANTEAMIENTO DEL PROBLEMA}

La enseñanza de la Matemática en la escuela secundaria en la mayoría de los casos no considera la resolución de problemas. Según estudios realizados por Aravena y otros (2008) en las clases predomina la ejercitación y el manejo de algoritmos y no se enfatiza la vinculación con problemas del mundo real y de las ciencias.

Los resultados de las evaluaciones de la Calidad Educativa en nuestro país dieron cuenta del estado de la educación secundaria. La experiencia en Facultades donde la Matemática se considera de carácter instrumental nos lleva a pensar que en el aprendizaje de la Matemática entrega una visión artificial del conocimiento, ya que no se comunica adecuadamente el saber científico, no se impulsa el proceso investigativo y se accede en forma esquemática y restringida a una imagen, no siempre fidedigna del mundo real.

En los primeros años de la universidad y aún en carreras en la que la Matemática se considera asignatura de carácter instrumental se plantea una alternativa entre la Matemática organizada de acuerdo con una concepción euclidiana, basada principalmente en la estructuración lógica, y la posibilidad de hacer de la intuición el motor de la construcción disciplinar, incluso de los propios procesos de formalización. En relación con esta segunda posibilidad se destaca el valor del Proyecto como eje del aprendizaje. Esta posición se basa en la existencia de dificultades en la comprensión de los conceptos matemáticos, desajustes entre los Significados Institucionales y el Significado Personal construido por el alumno y la posibilidad de diseñar algunas metodologías alternativas a la enseñanza tradicional en la Universidad que faciliten el aprendizaje.

\section{MARCO TEÓRICO}

El aprendizaje que inducimos en nuestra tarea de enseñar tiende, por lo general, a facilitar nuevas formas de proceder y de vivenciar. Ello exige la construcción de conceptos que se traducen en nuevos contenidos del quehacer y del pensamiento, los que se deben concretar en un saber a partir del cual el alumno sea capaz de juzgar y actuar correctamente ante nuevas situaciones. Una institución (escolar o de otro tipo) dirá que un sujeto comprende el significado de un objeto, o que ha captado el significado de un concepto, si dicho sujeto es capaz de realizar las distintas prácticas prototípicas que configuran el significado de dicho objeto en la institución (Godino, 2003)

Consideramos que un aprendizaje significativo es el que tiene en cuenta o atribuye un papel clave a la interacción social, la cooperación, el discurso, la comunicación y también a la interacción del sujeto con las situaciones-problemas (Godino, 2002)

En este sentido, en el trabajo que presentamos se considera el Proyecto como un dispositivo didáctico matemático esencial para el desarrollo del contrato didáctico y se muestra una experiencia de aplicación en el área de la Estadística.

In: M.A. Sorto (Ed.), Advances in statistics education: developments, experiences and assessments. Proceedings of the Satellite conference of the International Association for Statistical Education (IASE), July 2015, Rio de Janeiro, Brazil. 
Existen diferentes perspectivas vinculadas a la aplicación de modelos y a la modelización matemática con fines didácticos. Borba y Villarreal (2005) definen las siguientes:

1. La presentación de aplicaciones de conocimientos matemáticos en problemas reales.

2. El planteo, con fines de motivación, de un problema calificado como real que podrá ser resuelto a través del estudio de un cierto tópico matemático.

3. El trabajo con proyectos donde el tema y problemas asociados con el mismo son propuestos por el profesor.

4. El trabajo con proyectos donde el tema y los problemas asociados no son dados a priori, sino que los estudiantes son invitados a elegir el tema y, con la ayuda del profesor, a plantear problemas para ser resueltos.

Esta última perspectiva nos lleva a considerar el Proyecto como una instancia para que los alumnos desarrollen ideas matemáticas que puedan ser útiles para introducir el material didáctico que soporta desde la realidad el modelo que se desea desarrollar.

Los proyectos tienden a generar autonomía y responsabilidad en los alumnos así como a democratizar su acceso a los objetos de conocimiento, partiendo de las diferencias y privilegiándolas. (1988).

El método de proyectos responde a las etapas o fases del método didáctico que señala Aebli

Sanjurjo y Vera (1994) señalan que el ejercicio sin la construcción automatiza, desarrolla sólo la memoria mecánica, a partir de la cual los conocimientos se vuelven lábiles y rígidos, pero el ejercicio después de la construcción y la elaboración consolida y da consistencia.

Los elementos indispensables de todo proyecto, se pueden, pues, sintetizar en la respuesta a preguntas naturales:

\section{CUADRO 1 RELACIÓN ENTRE PREGUNTA Y RESPUESTA EN LA ELABORACIÓN DE UN PROYECTO}

\begin{tabular}{|c|c|}
\hline PREGUNTA & RESPUESTA \\
\hline ¿Por qué? & Fundamentación \\
¿Para qué? & Finalidad \\
¿A quiénes? & Beneficiarios \\
¿Cómo? & Acciones \\
¿Con qué? & Recursos \\
¿Quiénes? & Responsables \\
¿Cuándo? & Tiempo \\
\hline
\end{tabular}

Fuente: Álvarez, S. Proyectos integrados en el aula. (1990)

El objetivo es que, a partir de la reflexión sobre la propia práctica, se generan nuevas alternativas de trabajo que signifiquen una optimización de la propuesta pedagógico-didáctica de la/s disciplina/s en cuestión.

\section{CRITERIOS DE EVALUACIÓN DE LOS PROYECTOS}

Para Menin y Sanjurjo (1986), la evaluación de proyectos no será un acto único y final, sino un proceso continuo e integral, es decir que comienza desde el principio del proceso de aprendizaje y se ensambla continuamente con todos los otros aspectos de dicho proceso. Se evaluarán entonces, objetivos, contenidos y actividades. Entre los objetivos específicos se tratará de integrar tanto los que se refieren al área intelectiva (conocimientos y procesos intelectuales), como los referidos al logro de determinadas actitudes. La evaluación de actividades cobra importancia porque a través de ellas se pueden observar mejor los pasos seguidos para acceder a los contenidos y para lograr los restantes objetivos. 
Si el alumno está motivado para aprender, la evaluación surgirá como una necesidad y no como una imposición. Se infiere entonces otro principio básico de un nuevo sistema evaluativo: la participación del alumno en la evaluación de su propio trabajo.

\section{LA ELECCIÓN DEL TEMA}

El punto de partida para la definición de un Proyecto de trabajo es la elección del tema. Según Hernández y Ventura (1998), en cada situación esta elección adopta características diferentes. El alumno parte de sus experiencias anteriores, de la información que tiene sobre los proyectos ya realizados o en proceso de elaboración por otros grupos. Tanto el profesor como el alumno deben preguntarse sobre la necesidad, relevancia, interés u oportunidad de trabajar un determinado tema.

\section{¿POR QUÉ UN APRENDIZAJE DE LA ESTADÍSTICA BASADA EN PROYECTOS?}

En lugar de introducir los conceptos y técnicas descontextualizadas, o aplicadas únicamente a problemas tipo, difíciles de encontrar en la vida real, se trata de presentar las diferentes fases de una investigación estadística: planteamiento de un problema, decisión sobre los datos a recoger, recogida y análisis de datos y obtención de conclusiones sobre el problema planteado.

Anderson y Loynes (1987), señalan que la estadística es inseparable de sus aplicaciones y su justificación final es su utilidad en la resolución de problemas externos a la propia estadística.

Por otro lado, hay que diferenciar entre conocer y ser capaz de aplicar un conocimiento. La habilidad para aplicar los conocimientos estadísticos es frecuentemente mucho más difícil de lo que se supone, por qué requiere no sólo conocimientos técnicos (tales como preparar un gráfico o calcular un promedio), sino también conocimientos estratégicos (saber cuándo hay que usar un concepto o gráfico dado). Los problemas y ejercicios de los libros de texto sólo suelen concentrarse en los conocimientos técnicos. Los proyectos se conciben como verdaderas investigaciones, donde tratamos de integrar la Estadística dentro del proceso más general de investigación. Deben escogerse con cuidado, ser realistas (incluso cuando sean versiones simplificadas de un problema dado) abiertos y apropiados al nivel del alumno.

\section{LA TRAYECTORIA DIDÁCTICA}

La trayectoria didáctica en cada proyecto de Veterinaria una vez seleccionado el tema responde a las preguntas del NCTM (2000) en la siguiente forma:

1. ¿Cuál es mi problema? ¿Qué quiero probar?

La respuesta a esta pregunta requiere una ubicación del lugar de investigación, de una descripción de las instalaciones y de aquellos datos relativos al contexto que permitan enmarcarlo con precisión.

2. ¿Necesito datos? ¿Cuáles? ¿Qué tengo que medir u observar o preguntar?

La respuesta a esta pregunta exige el conocimiento del proceso de trabajo en el establecimiento o lugar elegido.

3. ¿Cómo encontrar los datos? ¿Qué hacer con ellos? ¿Cómo puedo obtenerlos?

Para dar respuesta a estas preguntas los alumnos analizan previamente trabajos análogos en el tema ya publicados.

4. ¿Cómo recoger los datos? ¿Observación, encuestas, experimentaciones?

5. ¿Qué hacer con los datos?

A través de la información se organizarán los datos para un análisis descriptivo de ella.

6. ¿Qué significan estos resultados en la práctica? ¿Cuál es su trascendencia en relación al problema planteado?:

Esta es la pregunta clave que da lugar a la comprensión de temas de estadística descriptiva y la necesidad de su interpretación en términos del problema.

\section{LA PUESTA EN MARCHA DE LOS PROYECTOS 2014 EN LA FACULTAD DE CIENCIAS VETERINARIAS. U.N.R.}

La experiencia se desarrolló durante el año 2014 en las cuatro comisiones en las que se dicta la asignatura Bioestadística, en la Facultad de Ciencias Veterinarias. 
Al comenzar el dictado de la asignatura se les explicó a los alumnos la metodología de trabajo: las clases serían teórico-prácticas, donde una hora semanal se dedicaría al seguimiento del proyecto elegido por cada grupo de tres alumnos quienes se agruparían por afinidad con el tema con el cual trabajarían. La aprobación del proyecto sería requisito para acceder al examen final en condición de alumno regular. En el proyecto se evaluarán los temas correspondientes a Estadística Descriptiva.

Consideramos que el momento que demandó más tiempo y esfuerzo fue la elección del tema y la búsqueda de la información y de datos sobre éste, ya que los alumnos debieron recabar datos en las distintas cátedras de la Facultad, en revistas científicas de la Biblioteca de la Facultad, en Internet o en trabajos de investigación facilitados por otros veterinarios.

Se realizó con cada grupo el análisis de los posibles temas del proyecto y luego se dividieron los temas de Estadística Descriptiva que se incluirían en el proyecto. Por cada tema se presentaron cuatro momentos de análisis del avance de los proyectos, de esta manera se corrigieron los errores en forma conjunta con los alumnos del grupo a través de la discusión y argumentación de los pasos y procedimientos realizados por los alumnos en el tema. Así, los alumnos pudieron superar sus dificultades y mejorar en forma continua sus propuestas. Se insistió en las exigencias de argumentación ya que fue una de las falencias que se preveían por experiencias anteriores en la práctica docente. Además, para fortalecer esta capacidad realizamos una puesta en común mensual, donde cada grupo comentaba su trabajo y hacía una reflexión personal sobre sus avances o retrocesos en el proyecto. Estos momentos permitieron a los alumnos exponer sus ideas, argumentar sobre sus trabajos y prepararse para la evaluación final del proyecto.

Las puestas en común mensuales fueron evaluadas por los mismos alumnos del grupo utilizando la estrategia didáctica de la metacognición y su opinión fue corroborada en la mayoría de las veces por la docente de cada comisión. La puesta en común final de cada grupo fue evaluado por todos los docentes de la cátedra.

Por cada Proyecto se consigna en el cuadro que sigue de doble entrada, la puesta en juego de los procesos que garantizan el aprendizaje de cada tema de la Unidad Didáctica elegida, en cuatro momentos del mismo.

\section{CUADRO 2 PROTOCOLO DE EVALUACIÓN DE UNA TRAYECTORIA DIDÁCTICA}

\begin{tabular}{|c|c|c|c|c|}
\hline Grupo $N^{\circ}$ & \multirow[b]{2}{*}{ Momentos } & \multicolumn{3}{|c|}{ Estadística descriptiva. } \\
\hline Integrantes & & $\begin{array}{l}\text { Cuadros y } \\
\text { gráficos. }\end{array}$ & $\begin{array}{l}\text { Medidas de } \\
\text { posición. }\end{array}$ & $\begin{array}{l}\text { Medidas de } \\
\text { dispersión y } \\
\text { asimetría. }\end{array}$ \\
\hline \multirow{4}{*}{ Comprensión conceptual } & $1^{\mathrm{o}}$ & & & \\
\hline & $2^{\circ}$ & & & \\
\hline & $3^{\circ}$ & & & \\
\hline & $4^{\circ}$ & & & \\
\hline \multirow{4}{*}{$\begin{array}{l}\text { Comprensión } \\
\text { procedimental }\end{array}$} & $1^{\mathrm{o}}$ & & & \\
\hline & $2^{\circ}$ & & & \\
\hline & $3^{\circ}$ & & & \\
\hline & $4^{\circ}$ & & & \\
\hline \multirow{4}{*}{$\begin{array}{l}\text { Contextualización del } \\
\text { problema }\end{array}$} & $1^{\circ}$ & & & \\
\hline & $2^{\circ}$ & & & \\
\hline & $3^{\circ}$ & & & \\
\hline & $4^{\circ}$ & & & \\
\hline \multirow{4}{*}{$\begin{array}{l}\text { Formulación y } \\
\text { comunicación en } \\
\text { terminología estadística }\end{array}$} & $1^{\mathrm{o}}$ & & & \\
\hline & $2^{\circ}$ & & & \\
\hline & $3^{\circ}$ & & & \\
\hline & $4^{\circ}$ & & & \\
\hline \multirow{4}{*}{$\begin{array}{l}\text { Validación de lo realizado. } \\
\text { Expresión de conclusiones } \\
\text { en términos del problema. }\end{array}$} & $1^{\circ}$ & & & \\
\hline & $2^{\circ}$ & & & \\
\hline & $3^{\circ}$ & & & \\
\hline & $4^{\circ}$ & & & \\
\hline
\end{tabular}




\section{Fuente: Elaboración propia}

En cuanto al rendimiento académico en términos de regularización de la asignatura, se presentan a continuación la relación entre alumnos que cursaron la asignatura y alumnos que alcanzaron la regularidad por la aprobación del Proyecto.

CUADRO 3 RESULTADOS DEL RENDIMIENTO ACADÉMICO SEGÚN COMISIÓN Y SITUACIÓN DE CURSADA.

\begin{tabular}{|c|c|c|c|c|c|c|}
\hline \multicolumn{4}{|c|}{ Alumnos que cursaron } & \multicolumn{3}{c|}{ Alumnos que aprobaron el proyecto } \\
\hline Comisión & $\mathbf{1}^{\mathbf{o}} \mathbf{v e z}$ & Recursantes & TOTAL & $\mathbf{1}^{\mathbf{0}} \mathbf{v e z}$ & Recursantes & TOTAL \\
\hline A & 45 & 20 & 65 & 40 & 18 & 58 \\
\hline B & 36 & 12 & 48 & 31 & 10 & 41 \\
\hline C & 37 & 11 & 48 & 34 & 7 & 41 \\
\hline D & 49 & 21 & 70 & 44 & 14 & 58 \\
\hline Total & 167 & 64 & 231 & 149 & 49 & 198 \\
\hline
\end{tabular}

Fuente: Datos obtenidos por la Cátedra de Bioestadística - Cursada 2014

Los porcentajes de aprobación final se consideraron muy satisfactorios. Los alumnos que no regularizaron la asignatura fueron aquellos que no demostraron compromiso a lo largo del desarrollo del proyecto o que al comenzar se desalentaron ante el trabajo de búsqueda de información.

Todos los proyectos que continuaron fueron exitosamente substanciados.

El cambiar de metodología permitió que los alumnos recursantes por $1^{\circ}, 2^{\circ}, 3^{\circ}$ y hasta $4^{\circ}$ vez se comprometieran con el estudio hasta lograr la aprobación de la asignatura con la defensa de su proyecto y el examen final.

El porcentaje de alumnos que regularizaron la asignatura superó en el año 2014 el 80\%, hecho muy positivo ya que el porcentaje anual de regularización en los últimos diez años fue del $60 \%$ o menor.

A manera de resumen, presentamos a continuación el porcentaje de alumnos que regularizaron la asignatura por comisión, es decir, aprobaron el proyecto.

\section{CUADRO 4 PORCENTAJE DE ALUMNOS REGULARES SEGÚN COMISIÓN}

\begin{tabular}{|c|c|}
\hline Comisión & \% de alumnos regulares \\
\hline A & 89 \\
\hline B & 85 \\
\hline C & 85 \\
\hline D & 83 \\
\hline
\end{tabular}

Fuente: Datos obtenidos por la Cátedra de

Bioestadística - Cursada 2014

El análisis anterior es un indicio importante de que el método de proyectos mejora sustancialmente la asimilación de diversos contenidos estadísticos. Los proyectos presentados incluyeron temas de Estadística descriptiva, como primer avance de análisis.

Al aplicar el método de proyectos en las distintas comisiones de Veterinaria se observa que a pesar de aumentar notablemente los porcentajes de alumnos aprobados, la "formulación y 
comunicación en terminología estadística" y "la validación de lo actuado y la expresión de conclusiones en términos del problema", continúan siendo los más ausentes en la construcción del concepto. A pesar de esto, con la aplicación del método de proyectos, se observan resultados satisfactorios en el rendimiento académico.

\section{CONCLUSIÓN}

El trabajar con el Método de Proyecto ha facilitado la creación de un ambiente de aprendizaje que afianza los conocimientos de Estadística Descriptiva, invitando a los alumnos a formular preguntas y procurar explicaciones. Además, promueve y desarrolla la formación de individuos socialmente activos.

Consideramos que el fundamento teórico y los resultados satisfactorios de la experiencia concretada en la Facultad de Ciencias Veterinarias (U.N.R.) constituyen un aporte metodológico que puede disminuir la incidencia de los factores que afectan negativamente (desajustes) la construcción del significado y del sistema de prácticas significativas en el proceso de enseñanza y aprendizaje de los temas de Estadística Descriptiva.

\section{BIBLIOGRAFÍA}

Aebli, H. (1988). Doce Formas Básicas de Enseñar. Madrid: Narcea.

Álvarez, S. (1990). Proyectos integrados en el aula. Buenos Aires: Kapelusz.

Anderson, C. W. y Loynes, R. M. (1987). The teaching of practical statistics. Nueva York: Wiley.

Aravena, M., Camaño, C. y otros (2008). Modelos Matemáticos a través de proyectos. Revista Latinoamericana de Investigación en Matemática Educativa, 11(1).

Borba, M. y Villarreal, M. (2005) Humans-with-media and the Reorganization of Mathematical Thinking: information and communication technologies, modeling, experimentation and visualization. Mathematics Education Library. V. 39. Springer-Verlag.

Godino, J. D. (2002). Un enfoque ontológico y semiótico de la cognición matemática. Recherches en Didactiques des Mathematiques, 22(2/3).

Godino, J. D. (2003). Teoría de las Funciones Semióticas en Didáctica de las Matemáticas. Universidad de Granada. Departamento de Didáctica de la Matemática. Disponible en: http://www.ugr.es/local/jgodino/funciones-semióticas/monografiatfs.pdf.

Graham, A. (1987). Statistical investigations in the secondary school. Cambridge: The Open University Centre for Mathematics Education.

Hernández, F. y Sancho, J. M. (1989). Para enseñar no basta con saber la asignatura. Barcelona: Laia.

Hernández, F. y Ventura, M. (1998). La organización del currículum por proyectos de trabajo. Barcelona: Graó

Lucarelli, E. A. y Correa, E. J. (1996). ¿Cómo generamos proyectos? Buenos Aires: Santillana.

Menin, O. y Sanjurjo, L. (1986). La evaluación del proceso de aprendizaje. Rosario: Irice. Conicet/ Universidad Nacional de Rosario.

N.C.T.M. (2000). Principles and standards for school mathematics. Reston, VA; N.C.T.M. Disponible en: http://standards.nctm.org/

Sanjurjo, L. y Vera, M. T. (1994). Aprendizaje significativo y enseñanza en los niveles medio y superior. Rosario: Homo Sapiens. 